\title{
Anatomía de la Cabeza Femoral Humana: Consideraciones en Ortopedia, Parte I. Anatomía y Relaciones Antropométricas del Fémur Proximal
}

\author{
Anatomy of the Human Femoral Head: Orthopedic Issues, Part I. \\ Proximal Femur Anatomy and Anthropometric Measures
}

*Raúl Álvarez San Martín \& **José Antonio Velutini Kochen

\begin{abstract}
ÁLVAREZ, S. M. R. \& VELUTINI, K. J. A. Anatomía de la cabeza femoral humana: consideraciones en ortopedia, parte I. Anatomía y relaciones antropométricas del fémur proximal. Int. J. Morphol., 28(2):427-431, 2010.

RESUMEN: Las variantes antropométricas son aspectos que han servido como sustento científico para el entendimiento de la vasta patología que afecta a la articulación coxofemoral y para el desarrollo tanto de implantes de reemplazo articular como de técnicas de reconstrucción ósea. En esta revisión se planea exponer los aspectos morfológicos macroscópicos de la cabeza femoral en relación al extremo proximal del fémur, para describir las características anatómicas y funcionales que hacen de ésta, uno de los sitios anatómicos de mayor importancia en la cirugía ortopédica.
\end{abstract}

PALABRAS CLAVE: Cabeza femoral; Fémur proximal; Variantes anatómicas; Macroestructura; Ortopedia.

\section{INTRODUCCIÓN}

El conocimiento de la anatomía de la cabeza femoral desde su desarrollo hasta sus modificaciones patológicas en la edad adulta ha logrado identificar aspectos causantes de muchas enfermedades que afectan a la articulación coxofemoral. Esto contribuye al diseño de implantes artificiales con el objeto de reconstituir su anatomía y función. Por tal motivo en este artículo se plantea actualizar al lector con algunos aspectos de la macroestructura de la cabeza femoral en relación al fémur proximal.

\section{Anatomía y antropometría del fémur proximal.}

Anatomía. La cabeza femoral normalmente tiene forma redondeada, con superficie lisa, representa los dos tercios de una esfera de 20 a $25 \mathrm{~mm}$ de radio y está orientada oblicuamente hacia arriba, medialmente y hacia adelante (Latarjet \& Ruiz-Liard, 1999). Está recubierta por cartílago hialino que se adelgaza hacia la periferia hasta estar ausente en la fóvea de la cabeza femoral, donde se va a insertar el ligamento de la cabeza del fémur ( Soames, 1988 y Miralles \& Puig, 1998). Esta fóvea se localiza en el cuadrante posteroinferior de la cabeza femoral en el $94 \%$ de los casos, siendo algo mayor en los hombres (Gutiérrez-Carbonel et al., 1991). Está formada por hueso compacto en la parte central con condensación subcondral en la periferia (Pablo \& Domínguez, 2002). La fóvea de la cabeza del fémur es oblonga y está orientada oblicuamente de superior a posteroinferior (esto acomoda la parte proximal del ligamento de la cabeza del fémur cuando está tenso, según Bardakos \& Villar, 2009).

Antropometría. La antropometría del fémur proximal se realiza a través de ángulos formados por los ejes mayores de sus estructuras anatómicas principales. En una vista AP del fémur, el ángulo cérvicodiafisiario está formado por el eje del cuello femoral que une a la cabeza femoral con el eje de la diáfisis femoral. Este también es denominado ángulo de inclinación o simplemente ángulo de Lanz. Sus valores

Profesor (Invitado) de Anatomía para Posgrado en Medicina. Departamento de Anatomía, Facultad de Medicina, Universidad Nacional Autónoma de México, UNAM. Ciudad de México, México.

** Profesor Adjunto del Curso de Ortopedia. Profesor de Anatomía para Posgrado en Medicina.

Facultad de Medicina, Universidad Nacional Autónoma de México, UNAM. Ciudad de México, México. 
se encuentran alrededor de los $125^{\circ}$ en el adulto (Miralles \& Puig), con unas variaciones de $115^{\circ}$ a $140^{\circ}$ según Kapandji (1988) o de $95^{\circ}$ a $135^{\circ}$, de acuerdo con Frankel \& Nordin (1980). La cifra aceptada es de $135^{\circ}$ y su método de medición radiográfica estandarizado ha sido descrito por Hoaglund \& Low (1980).

En una vista superior del fémur proximal, éste se encuentra angulado anteriormente con respecto del resto del fémur, característica denominada anteversión femoral (Rogers, 1931; Kinngsley \& Olmsted, 1948; Dunn \& Notley, 1952; Dunlap et al., 1953; Ryder \& Crane, 1953; Weiner et al., 1978; Hernández et al., 1981; Murphy et al., 1987; Nötzli et al., 2002. También es conocido como ángulo de torsión femoral o de declinación. Es propio de los humanos a consecuencia de la bipedestación (Solano et al., 2001). Está formado por las proyecciones sobre el plano transversal de un eje longitudinal del cuello femoral al centro de la cabeza femoral, intersectado por una línea tangencial al borde posterior de ambos cóndilos femorales (Muñoz, 1999), o bien, un eje del centro de la cabeza femoral a la base del cuello femoral intersectado por un eje transcondíleo (Murphy et al. (Fig. 1C). Su valor cambia con la edad desde $30^{\circ}-50^{\circ}$ a los 12 meses (Fabry et al., 1973; Shands \& Steele, 1958; Dunlap et al.; Budin \& Chandler) hasta su valor en la edad adulta de $10^{\circ}$ a $30^{\circ}$ (Miralles \& Puig), con variaciones de $12^{\circ}$ y $15^{\circ}$ (Frankel \& Nordin).

Una anteversión aumentada conlleva a una rotación medial de la cadera equivalente a una deformidad rotacional medial. Si la anteversión está disminuida (el fémur proximal angulado posteriormente), se conoce como retroversión y condiciona a un aumento de la rotación lateral de la cadera.

A la anteversión y retroversión también se les conoce como antetorsión y retrotorsión respectivamente. En ocasiones se ocupan los términos de torsión femoral medial y torsión femoral lateral como sinónimos de antetorsión (anteversión) y retrotorsión (retroversión) respectivamente (Staheli, 2002).

$\mathrm{Al}$ fémur humano se le ha clasificado en grupos funcionales gracias a las variaciones anatómicas angulares (antropométricas). Estos grupos son conocidos como brevilíneos y longilíneos; siendo los primeros mejor adaptados para la carga y los últimos para la carrera (Kapandji). Los clasificados como longilíneos tienen ángulos de declinación e inclinación mayores (longilíneo $25^{\circ} / 125^{\circ}$ y brevilíneo $10^{\circ} / 115^{\circ}$ ) que los brevilíneos, así mismo una cabeza femoral mayor ( $>2 / 3$ de una esfera en el fémur longilíneo en comparación con $1 / 2$ de una esfera en el fémur brevilíneo) (Kapandji) (Fig. 1 recuadro B). También se han encontrado diferencias angulares y endósticas del fé- mur proximal entre varias poblaciones (Gómez \& Robles, 1995; Marshall et al., 2008) y entre el fémur derecho con respecto al izquierdo (Da Silva et al., 2003).

Relaciones cervico-cefálicas. En un estudio hecho por Toogood et al. (2009) varios ángulos de relación cervicocefálico se usaron como parámetros de descripción de variantes anatómicas. Para las variantes de traslación de la unión cervico-cefálica se crearon "off-sets" y coeficientes de éstos, con base en las mediciones descritas por Ito et al. (2001) y Siebenrock et al. (2004). El parámetro de la variante de rotación cervico-cefálica se hizo a través del ángulo entre la cicatriz fisiaria y el eje del cuello femoral, en vistas AP y Lateral. La variante de concavidad de la unión cervico-cefálica se realizó con la medición del ángulo alfa descrito por Nötzli et al.; y se crearon modificaciones. En la Fig. 1 recuadros A, B y C se muestran las variantes de posición anatómica de la cabeza femoral con respecto del fémur proximal.

Con base en estas mediciones se encontró de manera global, que para la muestra estudiada de 375 fémures de un banco de huesos, la cabeza femoral tendía a ser trasladada anteriormente e inferiormente, rotada en abducción y anteversión, así como a tener una concavidad mayor posteriormente e inferiormente. En cuanto a la traslación relacionada al género, se encontró que los hombres tendían un Offset más inferior que las mujeres, en cuanto a la rotación, los fémures de los hombres mostraban más abducción y anteversión que las mujeres y finalmente en cuanto a la concavidad, los hombres y los sujetos mayores de 50 años tenían menos concavidad en la unión cervico-cefálica anterior, y menor esfericidad de la cabeza femoral que las mujeres y los sujetos menores de 50 años.

Lateralización femoral. Otra relación anatómica de la cabeza femoral dentro del fémur proximal es la lateralización femoral o también llamada Offset femoral (Charles et al., 2004; Bourne \& Rorabeck, 2002; Charnley, 1979). Se mide en una vista radiográfica convencional AP de la cadera, con los miembros inferiores en rotación medial de $10^{\circ}$ a $15^{\circ}$ y se define como la distancia desde el centro de rotación de la cabeza femoral hacia una línea que bisecta el eje mayor longitudinal del fémur a nivel de la punta del trocánter mayor (Della Valle et al., 2005). Fig. 1. Radiografía. Esta medición radiográfica puede variar por el grado de rotación de la cadera y aumentar con el tamaño del fémur (Rubin, 1992).

Otra correlación anatómica reportada entre el Offset femoral y la forma endomedular propuesta por Dorr et al. (1993), fue un centro de rotación de la cadera más medializado y alto para las metáfisis en forma de pipa y más lateralizado e inferior para aquellos en forma de copa de 
champaña (Lecerf et al., 2009). Su valor promedio se encuentra en el rango de $41 \mathrm{~mm}$ a $44 \mathrm{~mm}$ (Rubin), con rangos reportados en $27 \mathrm{~mm}$ hasta $57 \mathrm{~mm}$ (Charles et al.; Hodge et al.

Los ángulos de inclinación y declinación influyen en el Offset femoral de manera anatómica y funcional. El ángulo cervico-diafisiario detemina el tamaño del Offset femoral anatómico, aspecto importante pues influye sobre el largo del cuello y del miembro. En cambio la anteversión femoral define el Offset fisiológico o funcional, porque el aumento de la anteversión femoral puede llevar a un desplazamiento posterior del trocánter mayor y a una disminución del brazo de palanca y la fuerza del músculo glúteo medio (Lecerf et al).

El conocimiento de estas variantes anatómicas es fundamental para el diseño de implantes ortopédicos que reemplazan el extremo proximal del fémur. Un aspecto impor- tante es reestablecer el centro de rotación de la cadera asentado en la cabeza femoral, ya que con esto se puede mantener la longitud del miembro intervenido y el balance entre las fuerzas de reacción, la de los músculos abductores (Noble et al., 1988), además de asegurar la estabilidad articular e incrementar el rango de movilidad (Fackler \& Poss, 1980; McGrory et al., 1995; Soong et al., 2004).

En conclusión, el conocimiento a profundidad de la anatomía de la cabeza femoral en particular, obliga a que los estudios anatómicos y observaciones clínicas a futuro estén enfocados a dilucidar métodos estandarizados de medición combinando la tecnología actual y en desarrollo. Lo anterior permitirá a su vez en el contexto médico, identificar otros aspectos clave en la patología que afecta a la cadera, así como a determinar los impactos biológicos, económicos y sociales, sobre los implantes ortopédicos desarrollados y las técnicas de reconstrucción ósea creados para dicha articulación.

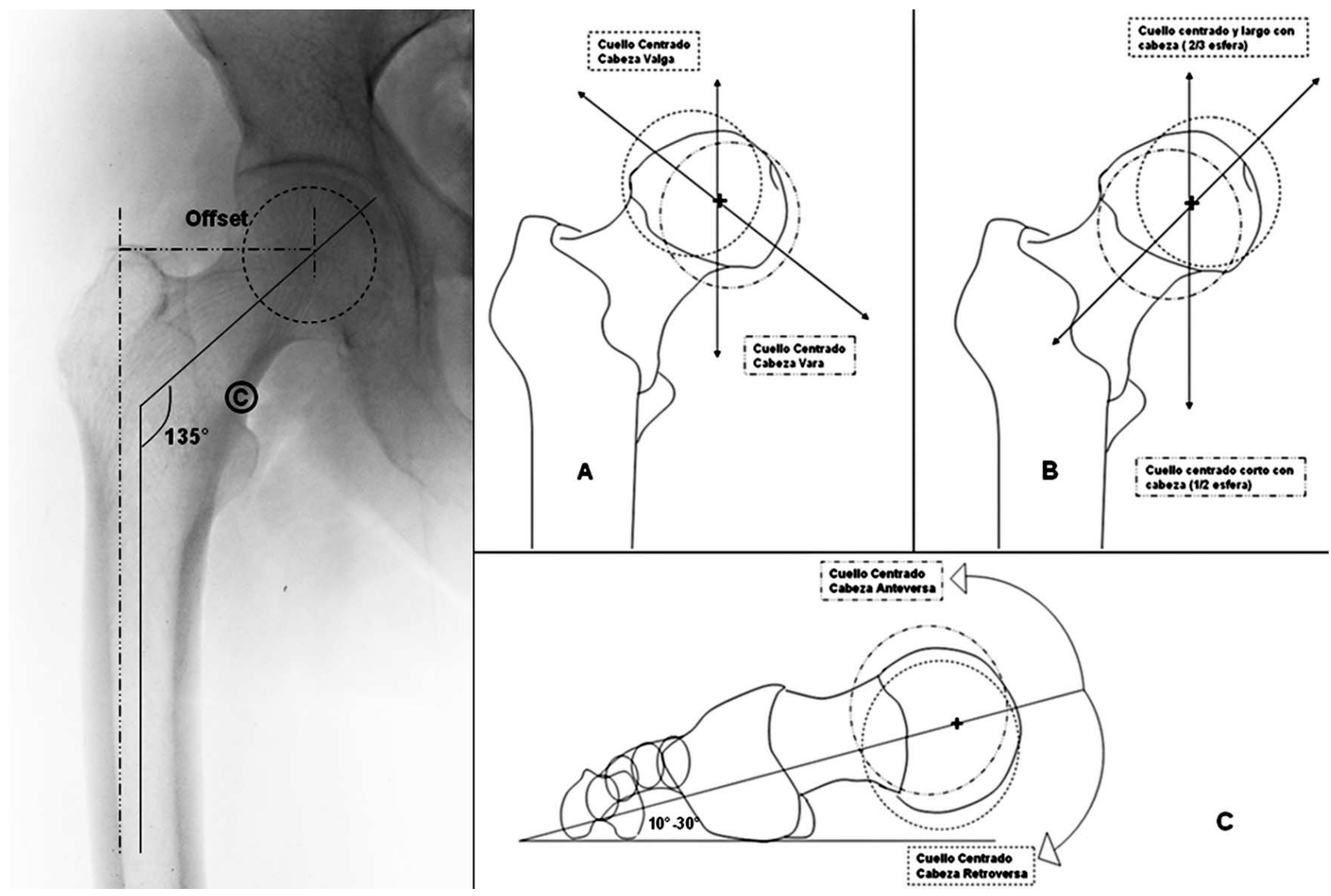

Fig. 1. Variantes Anatómicas de la cabeza femoral en el fémur proximal. Radiografía positiva en vista AP de la extremidad proximal del fémur. (-. - --) Líneas del Offset femoral. ( $\longrightarrow$ Líneas del ángulo cervicodiafisiario $=135^{\circ}$. (-----) Círculo que delimita el núcleo de la cabeza femoral. () Calcar. Izquierda Recuadros A, B y C. Representación de las variantes de posición anatómica de la cabeza femoral con respecto de ls extremidad proximal del fémur. Recuadro A Vista AP movimientos de varo y valgo. Recuadro B Vista AP movimientos de medialización y lateralización. Recuadro C Vista Superior movimientos de anteversión y retroversión. Centro de rotación de la cabeza femoral (+), ángulo de anteversión (declinación) del fémur proximal (10-30). 
ÁLVAREZ, S. M. R. \& VELUTINI, K. J. A. Anatomy of the human femoral head: orthopedic issues, part I. proximal femur anatomy and anthropometric measures. Int. J. Morphol., 28(2):427-431, 2010.

SUMMARY: The femoral head macroscopic and anthropometric anatomical variations are issues that have been used as scientific support for the understanding of the pathology which affects the hip joint and for the development of joint replacement implants and bone reconstruction techniques. The aim of this review is to expose the morphological macroscopic characteristics of the femoral head in relation to the proximal femur, in order to describe the functional and anatomical characteristics, which have made of this structure one of the most important anatomical areas in orthopedic surgery.

\section{KEY WORDS: Femoral head; Proximal femur; Anatomic variations; Macrostructure; Orthopedics.}

\section{REFERENCIAS BIBLIOGRÁFICAS}

Bardakos, N. V. \& Villar, R. N. The ligamentum teres of the adult hip. J. Bone Joint Surg. Br., 91(1):8-15, 2009.

Budin, E. \& Chandler, E. Measurement of femoral neck anteversion by a direct method. Radiology, 69(2):209-13, 1957.

Bourne, R. B. \& Rorabeck, C. H. Soft tissue balancing: The hip. J. Arthroplasty, 17(4):17-22, 2002.

Charles, M. N.; Bourne, R. B.; Davey, J. R.; Greenwald, A. S.; Morrey, B. F. \& Rorabeck, C. H. Soft-tissue balancing of the hip: the role of femoral offset restoration, J. Bone. Joint. Surg., 86(5):1078-88, 2004.

Charnley, J. Low friction principle. In: Low friction arthroplasty of the hip. Berlin, Springer-Verlag, 1979. pp.3-15.

Da Silva, V. J.; Oda, J. Y. \& Sant'Ana D. M. G. Anatomical aspects of the proximal femur of adult brazilians. Int. J. Morphol., 21(4):303-8, 2003.

Della Valle, G. A.; Padgett, D. E. \& Salvati, E. A. Preoperative planning for primary total hip arthroplasty. J. Am. Acad. Orthop. Surg., 13(7):455-462, 2005.

Dorr, L. D.; Faugere, M. C.; Mackel, A. M.; Gruen, T. A.; Bognar, B. \& Malluche, H. H. Structural and cellular assessment of bone quality of proximal femur. Bone, 14(3):231-42, 1993.

Dunlap, K.; Shands, A. R.; Hollister, L. C.; Gaul, J. S. \& Streit, H. A. A new method for determination of the torsion of the femur. J. Bone. Joint. Surg., 35-A(2):289-311, 1953.

Dunn, D. M. \& Notley, B. Anteversion of the neck of the femur: a method of measurement. J. Bone. Joint. Surg., 34 (2):181$6,1952$.
Fabry, G.; MacEwen, G. D. \& Shands, A. R. Torsion of the femur: a follow-up study in normal and abnormal conditions. J. Bone. Joint. Surg. Am., 55(8):1726-38, 1973.

Fackler, C. D. \& Poss, R. Dislocation in total hip arthroplasties. Clin. Orthop. Relat. Res., (151):169-78, 1980.

Frankel, V. H. \& Nordin, M. Basic Biomechanics of the Skeletal System. Philadelphia, Lea \& Febiger, 1980.

Gómez, G. F. \& Robles, U. A. Antropomorfometría endóstica del fémur proximal en población mexicana. Rev. Mex. Ortop. Traum., 9(2):59-70, 1995.

Gutiérrez-Carbonel, P.; Pachón-Ruíz, A.; Lizaur-Utrilla, A.; Sanchez del Campo, F. \& Anta Barrio, J. Estudio del área de la fóvea de la cabeza femoral. Rev. Ortop. Traum., 35(5):378-81, 1991.

Hernandez, R. J.; Tachdjian, M. O.; Poznanski, A. K. \& Dias, L. S. CT determination of femoral torsion. AJR. Am. J. Roentgenol., 137(1):97-101, 1981.

Hoaglund, F. T. \& Low, W. D. Anatomy of the femoral neck and head, with comparative data from Caucasians and Hong Kong Chinese. Clin. Orthop. Relat. Res., 152:10-6, 1980.

Hodge, W. A.; Andriacchi, T. P. \& Galante, J. O. A relationship between stem orientation and function following total hip arthroplasty. J. Arthroplasty., 6(3):229-35, 1991.

Ito, K.; Minka, M. A. 2nd.; Leunig, M.; Werlen, S. \& Ganz, R. Femoroacetabular impingement and the cam-effect: a MRIbased quantitative anatomical study of the femoral headneck offset. J. Bone. Joint. Surg. Br., 83(2):171-6, 2001.

Kapandji, I. A. Cuadernos de fisiología articular. 4. ed. Barcelona, Masson, 1988. 
Kingsley, P. C. \& Olmsted, K. L. A study to determine the angle of anteversion of the neck of the femur. J. Bone. Joint. Surg., 30(3):745-51, 1948.

Latarjet, M. \& Ruiz-Liard, A. Anatomía Humana. 3. ed. Buenos Aires, Panamericana, 1999. pp.774-88.

Lecerf, G.; Fessy, M.H.; Phillipot, R.; Massin, P.; Giraud, F.; Flecher, X.; Girard, J.; Mertl, P.; Marchetti, E. \& Stindel E. Femoral offset: anatomical concept, definition, assessment, implications for preoperative templating and hip arthroplasty. Orthop. Traumatol. Surg. Res., 95(3):2109, 2009.

McGrory, B. J.; Morrey, B. F.; Cahalan, T. D.; An, K. N. \& Cabanela, M. E. Effect of femoral offset on range of motion and abductor muscle strength after total hip arthroplasty. $J$. Bone. Joint. Surg. Br., 77(6):865-9, 1995.

Marshall, L. M.; Zmuda, J. M.; Chan, B. K. S.; Barrett-Connor, E.; Cauley, J. A.; Ensrud, K. E.; Lang, T. F. \& Orwoll E. S. Race and ethnic variation in proximal femur structure and BMD among older men. J. Bone. Min. Res., 23(1):121-30, 2008.

Miralles, R. C. \& Puig, M. Biomecánica clínica del aparato locomotor. Barcelona, Masson, 1998. pp. 211-24.

Muñoz, G. J. Atlas de Mediciones Radiográficas en Ortopedia y Traumatología. México D.F., Mac-GrawHill Interamericana, 1999. pp. 139-77.

Murphy, S. B.; Simon, S. R.; Kijewski, P. K.; Wilkinson, R. H. \& Griscom, N. T. Femoral anteversion. J. Bone. Joint. Surg., 69(8):1169-76, 1987.

Noble, P. C.; Alexander, J. W.; Lindahl, L. J.; Yew, D. T.; Granberry, W. M. \& Tullos, H. S. The anatomic basis of femoral component design. Clin. Orthop. Relat. Res., 235:148-65, 1988.

Nötzli, H. P.; Wyss, T. F.; Stoecklin, C. H.; Schmid, M. R.; Treiber, K. \& Hodler, J. The contour of the femoral headneck junction as a predictor of the risk of anterior impingement. J. Bone. Joint. Surg. Br., 84(4):556-60, 2002.

Pablo, de L. C. \& Domínguez, E. I. Tratamiento de las fracturas de huesos largos. En: Manual de Osteosíntesis, Barcelona, Masson, 2002. pp.88-97.

Rogers, S. P. A method for determining the angle of torsion of the neck of the femur. J. Bone. Joint. Surg. Am., 13:821-4, 1931.
Ryder, C. T. \& Crane, L. Measuring femoral anteversion: the problem and method. J. Bone. Joint. Surg. Am., 35(2):3218, 1953.

Rubin, P. J.; Leyvraz, P. F.; Aubaniac, J. M.; Argenson, J. N.; Esteve, P. \& De Roguin, B. The morphology of the proximal femur. A three-dimensional radiographic analysis. J. Bone. Joint. Surg., 74:28-32, 1992.

Shands, A. R. Jr. \& Steele, M. K. Torsion of the femur: a followup report on the use of the Dunlap method for its determination. J. Bone. Joint. Surg. Am., 40:803-16, 1958.

Siebenrock, K. A.; Wahab, K. H.; Werlen, S.; Kalhor, M.; Leunig, M. \& Ganz, R. Abnormal extension of the femoral head epiphysis as a cause of cam impingement. Clin. Orthop. Relat. Res., 418:54-60, 2004.

Soames, R. W. Sistema Esquelético. En: Anatomía de Gray. Madrid, Churchill Livingstone-Harcourt Brace, 1998. p.686.

Solano, L. A.; Brill, K. W.; Tey, P. M. \& Espiga, T. X. Desalineaciones torsionales de las extremidades inferiores. Implicaciones Clínico patológicas. En: Sociedad Española de Cirugía Ortopédica y Traumatología SECOT Monografías II. Barcelona, Masson-Elsevier, 2001.

Soong, M.; Rubash, H. E. \& Macaulay, W. Dislocation after total hip arthroplasty. J. Am. Acad. Orthop. Surg., 12(5):31421, 2004.

Staheli, L.T. T. Pediatric Orthopedic Secrets. $2^{\text {nd }}$ Ed. Philadelphia, Hanley \& Belfus Inc., 2002.

Toogood, P. A.; Skalak, A. \& Cooperman, D. R. Proximal femoral anatomy in the normal human population. Clin. Orthop. Relat. Res., 467(4):876-85, 2009.

Weiner, D. S.; Cook, W. A.; Hoyt, Jr.; Oravec, C. E. \& Lawrence, R. Computed tomography in the measurement of the femoral anteversion. J. Comput. Assist. Tomogr., 2:293, 1979.

\section{Dirección para correspondencia: \\ Dr. Raúl Álvarez San Martín \\ Calle B. Manzana X. No.19 Col. Educación. CP 04400 México D. F. Del. Coyoacán. \\ Ciudad de México MÉXICO}

Email: alvarez_sanmartin@yahoo.com.mx

Recibido : 16-01-2010

Aceptado: 22-03-2010 
